$\mathrm{BMC}$

Medical Imaging

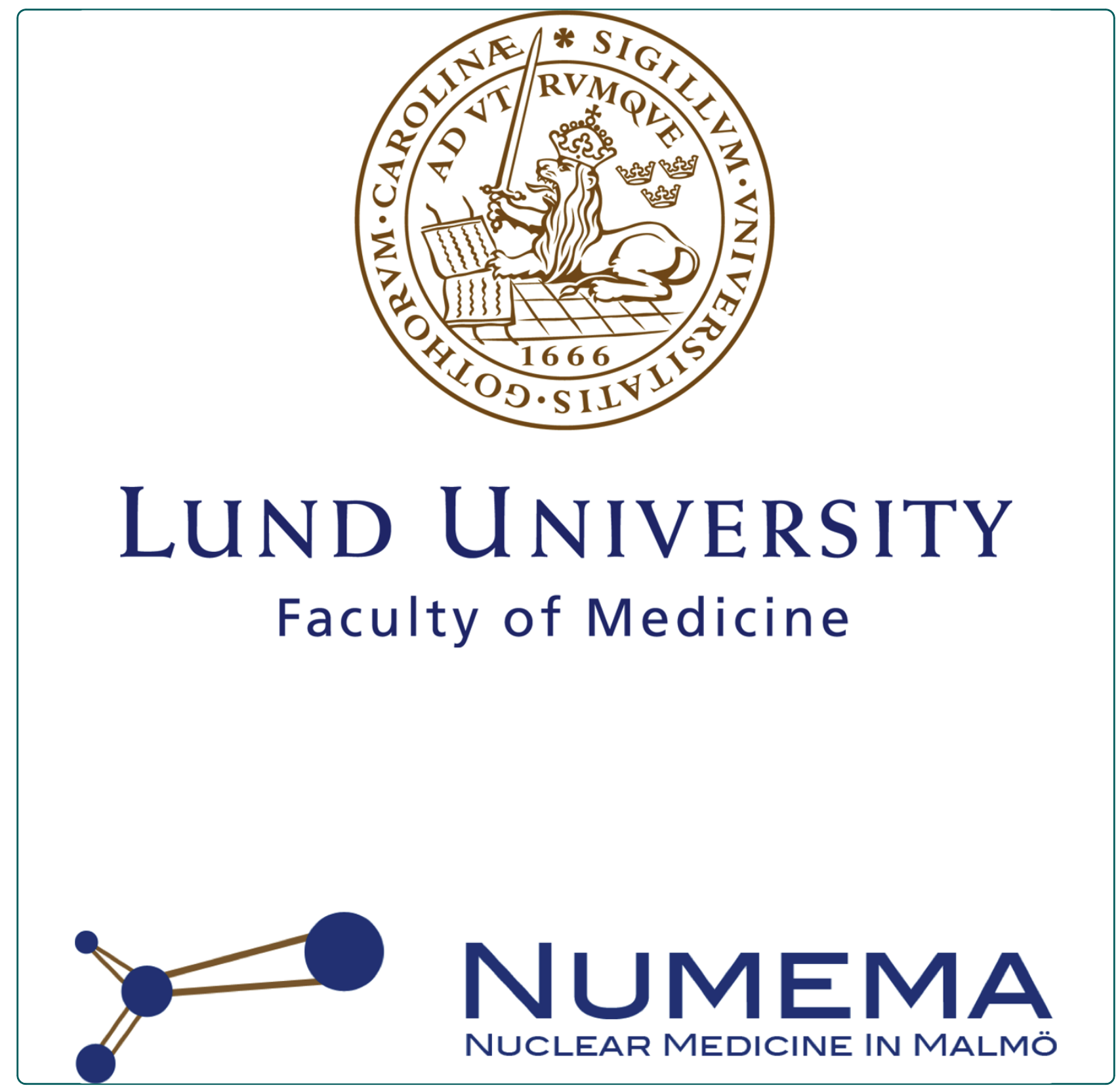

Semi-automatic analysis of standard uptake values in serial PET/CT studies in patients with lung cancer and lymphoma

Ly et al. 


\title{
Semi-automatic analysis of standard uptake values in serial PET/CT studies in patients with lung cancer and lymphoma
}

\author{
John Ly' ${ }^{1}$, Sabine Garpered ${ }^{1}$, Peter Höglund ${ }^{2}$, Eskil Jönsson'1, Sven Valind', Lars Edenbrandt ${ }^{1,3}$ and Per Wollmer ${ }^{1,4^{*}}$
}

\begin{abstract}
Background: Changes in maximum standardised uptake values (SUV $V_{\text {max }}$ ) between serial PET/CT studies are used to determine disease progression or regression in oncologic patients. To measure these changes manually can be time consuming in a clinical routine. A semi-automatic method for calculation of SUV max in serial PET/CT studies was developed and compared to a conventional manual method. The semi-automatic method first aligns the serial PET/CT studies based on the CT images. Thereafter, the reader selects an abnormal lesion in one of the PET studies. After this manual step, the program automatically detects the corresponding lesion in the other PET study, segments the two lesions and calculates the SUV max in both studies as well as the difference between the SUV $V_{\max }$ values. The results of the semi-automatic analysis were compared to that of a manual SUV max $_{\text {analysis using a }}$ Philips PET/CT workstation. Three readers did the SUV max readings in both methods. Sixteen patients with lung cancer or lymphoma who had undergone two PET/CT studies were included. There were a total of 26 lesions.

Results: Linear regression analysis of changes in $\mathrm{SUV}_{\max }$ show that intercepts and slopes are close to the line of identity for all readers (reader 1: intercept $=1.02, R^{2}=0.96$; reader 2 : intercept $=0.97, R^{2}=0.98$; reader 3 : intercept $=0.99, R^{2}=0.98$ ). Manual and semi-automatic method agreed in all cases whether $S U V_{\max }$ had increased or decreased between the serial studies. The average time to measure SUV $\max$ changes in two serial PET/CT examinations was four to five times longer for the manual method compared to the semi-automatic method for all readers (reader 1: 53.7 vs. 10.5 s; reader 2: 27.3 vs. 6.9 s; reader 3: 47.5 vs. 9.5 s; $p<0.001$ for all).

Conclusions: Good agreement was shown in assessment of SUV $\max$ changes between manual and semi-automatic method. The semi-automatic analysis was four to five times faster to perform than the manual analysis. These findings show the feasibility of using semi-automatic methods for calculation of SUV $V_{\max }$ in clinical routine and encourage further development of programs using this type of methods.
\end{abstract}

Keywords: Image analysis, Radionuclide imaging, Quantification

\section{Background}

Changes in maximum standard uptake values $\left(\mathrm{SUV}_{\max }\right)$ and computed tomography (CT) tumour size in ${ }^{18} \mathrm{FDG}$ positron emission tomography (PET)/CT follow-up studies in oncologic patients are often assessed in tandem to determine disease progression or regression. RECIST criteria based on CT [1] are most frequently used, but criteria based on the uptake of ${ }^{18} \mathrm{FDG}$ are receiving increasing attention $[2,3]$. With this, the demand for quantitative

\footnotetext{
* Correspondence: per.wollmer@med.lu.se

'Department of Clinical Sciences, Skåne University, Malmö, Sweden Full list of author information is available at the end of the article
}

monitoring of SUV has grown as the indications for performing follow-up ${ }^{18}$ FDG PET/CT are expanding [4]. $\mathrm{SUV}_{\max }$ measurements are more readily reproducible than CT sizes in both pre- and post-treatment studies [5]. Currently available PET/CT software enables the use of tools that can determine $\mathrm{SUV}_{\max }$ by defining a ROI/VOI both manually and automatically within one examination. To our knowledge, none of the PET/CT manufacturers offer a software suite that can measure two matching uptakes in serial examinations automatically.

There are several studies on tumour delineation and quantification in PET/CT [6-12]. New quantitative

\section{C) Biomed Central}


approaches to evaluate treatment response have been suggested in some papers $[6,11]$. But differences in imaging parameters across PET centres have a negative effect on semi-automatic methods' performance [13,14]. Due to the increasing amount of examinations and the quantification that can be done, standardization of PET image acquisition and effective computer-aided quantification is warranted.

A common clinical setting for PET/CT is the evaluation of follow-up examinations after treatment or expectancy. Manual measurement of $S U V_{\text {max }}$ in single studies or changes in serial studies can be time consuming, making it difficult for clinicians and PET reviewers to follow-up all $\mathrm{SUV}_{\max }$ changes in serial studies in a comprehensive and effective way. Studies with semi-automatic quantification of $\mathrm{SUV}_{\max }$ changes in serial examinations suggest registration of images and SUV measurements are accurate and reliable $[6,11]$. Time saving qualities, or investigation of the reproducibility of using a semi-automatic approach, compared to manual reading of $\mathrm{SUV}_{\max }$ has not been elicited. The purpose of this study is to demonstrate these qualities using a semi-automatic method for calculation of $\mathrm{SUV}_{\max }$ of an abnormal lesion in serial studies when a region of interest (ROI) has been semi-automatically defined in the PET image.

\section{Materials and methods Patients}

Patients with lung cancer or lymphoma who had undergone two PET/CT studies between July 2008 and January 2010 at the Skåne University Hospital in Malmö, Sweden were included retrospectively.

There were ten lung cancer patients and six lymphoma patients. Diagnoses were verified with biopsy in all cases except for one patient on which the diagnosis was based on clinical findings. The patients had not been treated with chemotherapy or radiation therapy for at least 6 months prior to the baseline examination. The time from baseline examination to the follow-up examination varied between 31 and 150 days. Follow-up examinations were performed to evaluate tumour progression in five cases and therapy response in eleven cases.

Pathological lesions with sharp contrast to surrounding areas, no formation of a large conglomerate mass and presence in both studies were selected. 16 patients $(69 \%$ men, age $56 \pm 15$ (mean \pm SD)) with 26 pathological lesions were included. Each patient had one to four pathological lesions for manual and semi-automatic measurement. The study was approved by the Research Ethics Committee at Lund University.

\section{Scan data}

After 4-6 hours fast $4 \mathrm{MBq} / \mathrm{kg}$ of ${ }^{18} \mathrm{FDG}$ was given intravenously. The effective dose was 5-7 $\mathrm{mSv}$ for PET,
2-3 mSv for low-dose CT and 10-15 for diagnostic CT. Data were acquired $60 \mathrm{~min}$ after injection and the patients were scanned from head to the upper thigh with the use of an integrated PET/CT system (Philips Gemini TF). A diagnostic/low dose CT scan obtained with the use of a standard protocol, $150 \mathrm{mAs} /$ slice, $120 \mathrm{kV}$, a tube rotation time of $0.75 \mathrm{~s}$ per CT rotation, a pitch of 0.9 , and a slice thickness of $5 \mathrm{~mm}$ preceded the PET scan covering the identical transverse field of view (a 2 min emission scan per table position and 6-10 bed positions per patient).

The PET image data sets were reconstructed iteratively with segmented correction for attenuation with use of the CT data. CT images, PET images and co-registered images were displayed by means of Philips workstation and program. Reconstructed PET and CT images were also transferred to a customized software developed for semi-automatic measurements.

\section{SUV $_{\text {max }}$ measurements}

Three readers did manual and semi-automatic analysis of $\mathrm{SUV}_{\max }$ on 26 pathological lesions. One of the readers marked the 26 lesions in screenshots of coronal and transaxial PET images and these images were used during the study to secure that all three readers were measuring the same lesions with the manual and the semi-automatic method. The time to measure SUV $\mathrm{max}_{\max }$ manually and semiautomatically, after complete loading of the serial studies, was recorded on all pathological lesions individually.

\section{Manual method}

Manual measurements were done on a Philips PET/CT workstation (Philips Extended Brilliance Workspace, PET/CT Application Suite v1.5 K). The readers identified the lesions in both PET studies and used their most preferred software tool to determine the $S U V_{\text {max }}$. Readers reported that they used the free-floating SUV search tool and ROIs.

\section{Semi-automatic method}

A method to measure $\mathrm{SUV}_{\max }$ of a pathological lesion present in two studies from the same patient was developed. Transaxial PET and CT images from the two studies were used as input to the program. The two CT studies were automatically aligned by projecting the skeletons in the CT images on a $2 \mathrm{D}$ space. A rigid registration algorithm was used. CT images were reduced in size, blurred, thresholded and then overlaid to compare pixels with the formula: $\Sigma_{(i, j)} \operatorname{erf}(i, j)=\left|\operatorname{IM}_{1}(i, j)-\operatorname{IM}_{2}(i, j)\right|$

One of the images is repositioned and the formula is applied again to calculate the pixel error. The process is reiterated with less size reduction and blurring each time.

The reader marked an arbitrary pixel of a pathological lesion in one of the two PET studies. After this manual 
step the program automatically detects the corresponding lesion in the second PET study. The reader's mark in the first PET study has a corresponding location in the first CT study, provided that PET and CT images were correctly aligned from the PET/CT camera. The corresponding location in the second CT study was defined using the matching by the software and the corresponding location in the second PET study was defined.

The volumetric segmentation of the pathological lesions was made in the PET images. The reader's mark in the first PET study and the corresponding location in the second PET study were used as seed points for the segmentation using the graph-cut algorithm [15]. SUV$\max$ in the entire lesion was then calculated automatically and the segmentations were presented to the reader. Semi-automatic analysis was done on a standard laptop computer (Figure 1).

\section{Statistical analyses}

Intraobserver reproducibility was analysed with Intraclass correlation (ICC). A Bland-Altman analysis was used to assess the level of agreement between the two methods.

\section{Results}

The Bland-Altman analysis indicates that the $95 \%$ limits of agreement between manual and semi-automatic assessment ranged from -2.67 to 1.29 (reader 1 ); -3.39 to 2.29 (reader 2 ); -2.21 to 1.18 (reader 3 ). The two methods consistently provide similar measures for most of the tumours. Limits of agreement ranged from \pm 0.99 to \pm 1.41 across the readers. Manual and semi-automatic method agreed in all cases whether $S_{U V} V_{\max }$ had increased or decreased between the serial studies. The range of $\mathrm{SUV}_{\max }$ values for the manual method was 2.4 19.8 and for the semi-automatic method 2.6 - 21.4.
ICCs of $\mathrm{SUV}_{\max }$ readings between readers using the semi-automatic method in the first and second study were 1.00 (95\% CI, 1.00-1.00) and 0.94 (95\% CI, 0.89$0.97)$ respectively. ICC of SUV $\mathrm{Smax}_{\max }$ readings between readers using the manual method were 1.00 (95\% CI, $1.00-1.00)$ and 0.95 (95\% CI, 0.90-0.97) for the first and second study respectively. Almost-perfect reproducibility was thus obtained with both methods.

The average time to measure $\mathrm{SUV}_{\max }$ changes in two serial PET/CT examinations was significantly longer for the manual method compared to the semi-automatic method for all readers (Table 1). The semi-automatic method is up to 4 - 5 times faster compared to the manual method (Figure 2).

\section{Discussion}

Good agreement between the two methods was showed with the Bland-Altman analysis. The somewhat wider range of limits of agreement in reader $2( \pm 1.41)$ seems to be due to not finding the highest $S U V_{\max }$ with the manual method within some large tumours with high $\mathrm{SUV}_{\text {max }}$. This resulted in a greater difference comparing to the semi-automatic method. Both methods agreed whether the SUV $V_{\max }$ had increased or decreased in the follow-up examination. The results of this study indicate the feasibility of using semi-automatic method in serial examinations.

Like other studies $[5,6,9,10]$, the selection of tumours was limited to small-medium sized tumours, which were round, had a clear delineation on the CT scan and were not part of large conglomerates, which produce a "bleeding" effect on the PET image. The almost-perfect reproducibility obtained with both methods is likely dependent on the properties of the pathological sites. The finding supports the findings in previous studies $[5,6,9,10]$. Clinical reality provides many obstacles for semi-automatic

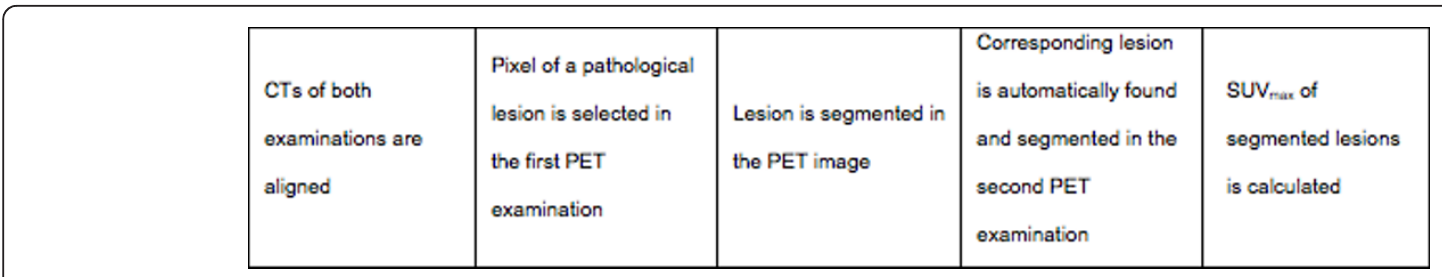

Figure 1 Flowchart of the semi-automatic process.

Table 1 Average time to measure changes in $\mathbf{S U V}_{\max }$ between two serial examinations for manual and semi-automatic methods and the difference between them

\begin{tabular}{lcclc}
\hline & Manual & Semi-automatic & Difference (95\% Cl) & $\boldsymbol{p}$-value \\
\hline Reader 1 & $53.7 \mathrm{~s}$ & $10.5 \mathrm{~s}$ & $43.2(36.4-49.9)$ & $<0.001$ \\
Reader 2 & $27.3 \mathrm{~s}$ & $6.9 \mathrm{~s}$ & $20.4(13.6-27.1)$ & $<$ \\
Reader 3 & $47.5 \mathrm{~s}$ & $9.5 \mathrm{~s}$ & $38.1(31.3-44.8)$ & 4 \\
\hline
\end{tabular}




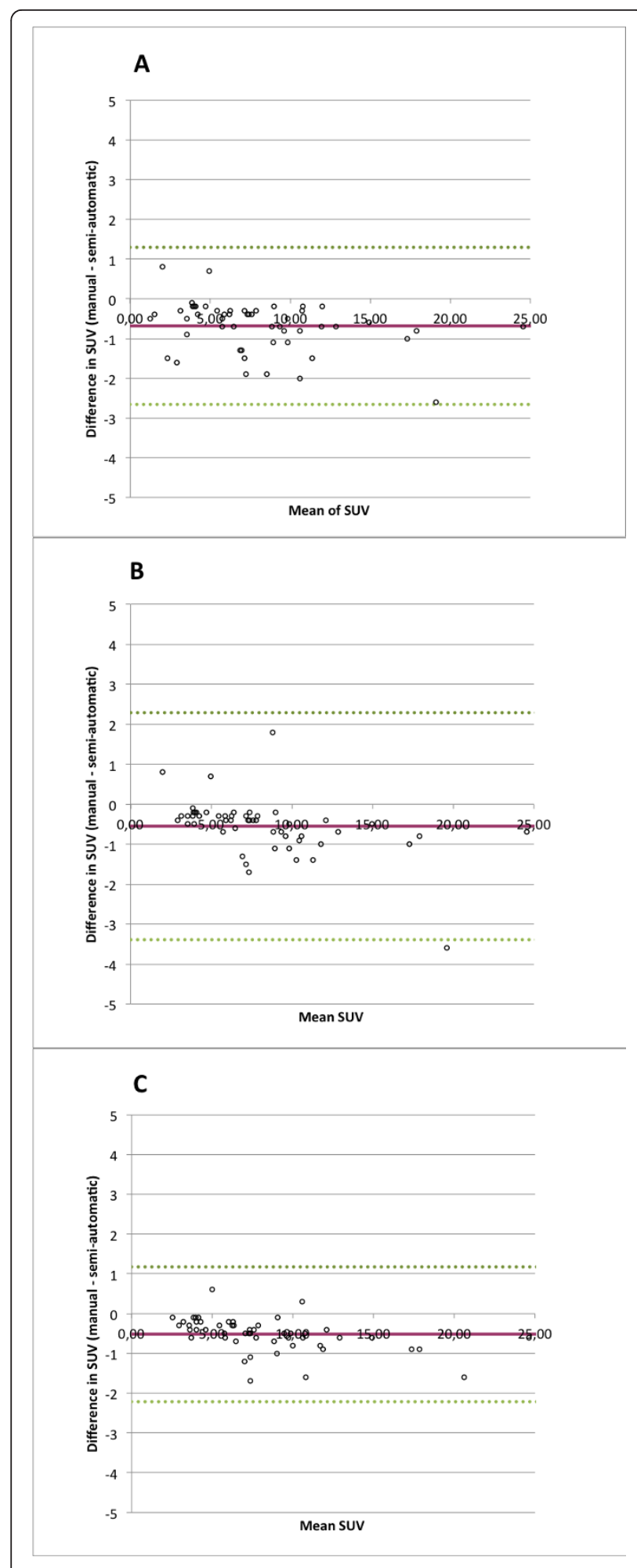

Figure 2 Bland-Altman analysis of SUV $\mathrm{max}_{\max }$ differences between both methods. Limits of agreement for $A:-2.67$ to 1.29 (reader 1); B: -3.39 to 2.29 (reader 2); C: -2.21 to 1.18 (reader 3) segmentation and it remains to be seen how pathological sites can be best segmented by algorithms no matter the form, size or location it has.

Readers were not instructed whether they should perform the semi-automatic segmentation in the first or second study. In the feedback after measuring with both methods, readers agreed it was easier to segment the correct tumour of both examinations by selecting the tumour on the examination, which had the smallest size. Should the readers have been instructed only to segment in the first or second scan regardless, the results may have shown poorer segmentation quality.

Segmentation in the PET image has more advantages than segmentation in CT. In the study it enabled segmentation of lymphoma in the liver and in locations where the lesion in CT was adjacent to soft tissue. Also, the segmentation tool used did not adequately segment the lesions in CT.

Manual delineation capabilities were intentionally left out of the program in order to test the accuracy of semi-automatic segmentation and in order to keep the time of analysis to a minimum.

$\mathrm{SUV}_{\text {max }}$ was chosen over $\mathrm{SUV}_{\text {mean }}$ due to clinical praxis at our hospital when assessing PET/CT examinations. Furthermore, research has shown $\mathrm{SUV}_{\max }$ measurements are readily reproducible between readers [5]. The program shows systematically higher $\mathrm{SUV}_{\max }$ compared with the manual method. This may be due to different filter settings between the PET/CT manufacturer and the program. It is unlikely that all readers have systematically missed the "true" $\mathrm{SUV}_{\max }$ with the program.

\section{Conclusions}

Good agreement was shown in absolute $\mathrm{SUV}_{\max }$ measurements between both methods. Almost-perfect reproducibility was seen between three readers using both semi-automatic and manual methods. Using semi-automatic method reduces time to calculate $S U V_{\max }$ by up to 5 times. The findings show feasibility of using semiautomatic calculation of SUV $\mathrm{max}_{\max }$ in serial studies and encourage further development of programs that accurately segments more complex pathological sites.

\section{Funding}

This research is supported by grants from ALF (all authors).

\section{Author details}

1'Department of Clinical Sciences, Skåne University, Malmö, Sweden. ${ }^{2}$ Competence Centre for Clin Research, Lund University, Lund, Sweden. ${ }^{3}$ Department of Molecular and Clinical Medicine, Sahlgrenska Academy, Gothenburg University, Gothenburg, Sweden. ${ }^{4}$ Clinical Physiology and 


\section{Authors' contributions}

$J \mathrm{~L}$ contributed to patient recruitment, did manual reading, took part in data analysis and wrote the first version of the manuscript. SG contributed to patient recruitment and did manual reading. $\mathrm{PH}$ contributed to the development of the semi-automatic method and to data analysis. EJ contributed to the development of the semi-automatic method and performed the programming. SV contributed to development of the semiautomatic method, patient recruitment and did manual reading. LE conceived of the study and contributed to the design of the study, to development of the semi-automatic method and to data analysis. PW contributed to the design of the study and to data analysis. All authors read and approved the final manuscript.

\section{Competing interests}

The authors declare that they have no competing interests.

Received: 10 August 2011 Accepted: 2 April 2012

Published: 2 April 2012

\section{References}

1. Eisenhauer EA, et al: New response evaluation criteria in solid tumours: revised RECIST guideline (version 1.1). Eur J Cancer 2009, 45(2):228-247.

2. Ben-Haim S, et al: 18F-FDG PET and PET/CT in the evaluation of cancer treatment response. J Nucl Med 2009, 50(1):88-99, Review.

3. Hicks RJ: Role of $18 \mathrm{~F}-$ FDG PET in assessment of response in non-small cell lung cancer. J Nucl Med 2009, 50(Suppl 1):31S-42S.

4. Blodgett TM, et al: PET/CT: Form and Function. Radiology 2007, 242(2):360-385, Review.

5. Jacene HA, et al: Assessment of interobserver reproducibility in quantitative 18-F-FDG PET and CT measurements of tumor response to therapy. J Nucl Med 2009, 50:1760-1769.

6. Gao X, et al: Computer-assisted quantitative evaluation of therapeutic responses for lymphoma using serial PET/CT imaging. Acad Radiol 2010, 17:479-488.

7. Tylski, et al: Comparative assessment of methods for estimating tumor volume and standardized uptake value in 18F-FDG PET. J Nucl Med February 2010, 51(2):268-276.

8. Wang, et al: Computer-assisted quantification of lung tumors in respiratory gated PET/CT images: phantom study. Med Biol Eng Comput 2010, 48:49-58.

9. Yan J, et al: Marker-controlled watershed for lymphoma segmentation in sequential CT images. Med Phys 2006, 33(7):2452-2460.

10. Yan J, et al: Automated matching and segmentation of lymphoma on serial CT examinations. Med Phys 2007, 34(1):55-62.

11. Fox, et al: Practical approach for comparative analysis of multilesion molecular imaging using a semiautomated program for PET/CT. J NuCl Med 2011, 52:1727-1732.

12. Hatt, et al: PET functional volume delineation: a robustness and repeatability study. Eur J Nucl Med Mol Imaging 2011, 38:663-672.

13. Cheebsumon, et al: Impact of [18F]FDG PET imaging parameters on automatic tumour delineation: need for improved tumour delineation methodology. Eur I Nucl Med Mol Imaging 2011, 38(12):2136-2144, ISSN 1619-7070, 12/2011.

14. Cheebsumon NO, et al: Effects of image characteristics on performance of tumor delineation methods: a test-retest assessment. J Nucl Med 2011, 52(10):1550-1558.

15. Boykov Yuri, et al: Interactive graph cuts for optimal boundary \& region segmentation of objects in N- D images. Computer Vision 2001, 1:105-112.

\section{Pre-publication history}

The pre-publication history for this paper can be accessed here: http://www.biomedcentral.com/1471-2342/12/6/prepub

doi:10.1186/1471-2342-12-6

Cite this article as: Ly et al:: Semi-automatic analysis of standard uptake values in serial PET/CT studies in patients with lung cancer and lymphoma. BMC Medical Imaging 2012 12:6

\section{Submit your next manuscript to BioMed Central and take full advantage of:}

- Convenient online submission

- Thorough peer review

- No space constraints or color figure charges

- Immediate publication on acceptance

- Inclusion in PubMed, CAS, Scopus and Google Scholar

- Research which is freely available for redistribution 